in Japan. In all these, as well as in the case of the hills near Freiburg mentioned by Dr. Wetterhan, the soil appears to be a porous detritus with a hard substratum. At St. Anton, as at Chamoanix, the hill-side sloped at an angle of about $50^{\circ}$, with a northern aspect, and in both cases and in Japan the phenomenon occurred in the autumn, a season often characterised, esp cially at high elevations, by cold nights and genial days. Dr. Koch calls it "smdethar" and "gans cigenthïmlich," and it is plainly not of common occurrence.

Dr. Koch's explanation of the phenomenon is virtually the same as had occurred to me, except that both he and Dr. Wetterhan appear to consider that the water was derived by absorption from a moist atmospherc. In none of the descriptions, however, is there any mention of what was one of the most striking features of the ice which I tried to describe, viz. its division into distinct layers, each layer being of uniform depth ; and this, showing as it docs that the crystallisation was interrupted, and not continuous, seems to make it more probable that the water was supplied from below. The cylindri-al perfora. tions were, no douht, caused by the presence of pebbles or small lumps of earth too dense to allow the ice-crystals to penetrate them, and too heavy to be pushed up. The layer of dust on the surface was much thinner in my case than in Dr. Koch's, which was no doubt due to accidental difference in the soil.

A friend in the country tells me that on a bright winter's day two or threc years ago he picked up a piece of a dead beechbranch which was covered with filamentous ice, such as is dcscribed by the Duke of Argyll and others in NATURe (vol, xxi. pp. 274, 302). He brought it home, and, having examined it, left it out in the sun, when the crystals of course soon vanished. Next morning, however, he was surprised to see that they had all reappeared as before. The water from the melting ice had again filled the pores of the wood, and ayain been extruded in the same crystalline form. Now, if the highest temperature to which they had been exposed during the day had been $32^{\circ} \mathrm{F}$., and a fresh supply of water had been afforded from any source to the wood, then neither would the ice have melted nor the water frozen ; until the temperature fell again at night, when a fresh formation of crystals would have taken place, which would have pushed up those previously existing, and the result would have been a formation similar to that described in my letter. It seems more probable, therefore, that the moistening took place from below, as I suggested.

Hampstead, December 20, 1884

R. Woodd Smith

\section{Lightning in the Tropics}

My experience confirms the remarks of Dr. Von Danckelman in NATURE (p. 127) respecting the little damage done by lightning in tropical climate:

In the plains of India at the commencement of the monsoon, storms occur in which the lightning runs like snakes all over the sky at the rate of three or four flashes in a second, and the thunder roars without a break for, frequently, one or wwo hours at a time. During twelve years' residence in India I heard of only two human beings and, I think, three buildings being struck, although in parts of Lower Bengal the population amounts to more than 600 in the square milc. I always attributed the scarcity of accidents to the great depth of the stratum of heated air next the ground lseeping the clouds at such a heigh that most of the flashe; pass from cloud to cloud, and very few reach the earth. This idea is supported by the fact that in the Himalayas, at 6000 feet or more above the sea, buildings and trees are frequently struck. I have secn more than a dozen pinetrees which had been injured by lightning on the top of one mountain between 8000 and 9000 feet high. In the Britisl Islands thunderstoms are said to be more dangerous in winter than in summer, and such a fact, if true, can be explained by the very thin stratum of air then intervening between the clouds and earih.

London, December 19,1884 J. J. MEYRICK

\section{An Unnoticed Factor in Evolution}

I AM surprised that the letter of Mr. Catchpool in NAlURE (vol. xxxi. p. 4) has remained unnoticed by your correspondents. His hypothesis that mutual stcrility may be the cause, not the result, of specific divergence, is, I think, quite in accordance with many observed facts. The buffalo and the ox, the sheep and the goat, have lived for ages side by side without, as far as I am aware, a hybrid between either of them having been produced. Mule or hinny hybrids between the horse and the ass are obtained easily, but the offspring is rarely fertile, so rare, that the British Consul at Granada told me, when I was there, that he had never known of a case, although in Spain mules exist in thousands. Amongst bovine animals many species produce hybrids which are apparently perfectly fertile; those between the Indian ox and the gayal, species of different genera, Bos and Bibos, are common, and their fertility is shown by the existence of numerous intermediate hybrids. There is living at the Zoological Gardens at the present time, a hybrid between the Indian ox, the gayal, and the bison, and, by her side, a hybrid between herself and a bison. The offspring of the cross between many species of ducks are perfectly fertile. 'This I have repeatedly seen in the case of the hylrids between the tufted duck and the pochard. I think there is another unno. ticed factor in evolution. The scent of animals plays an important part in their sexual relationships, and "sports" in this respect are as likely to occur as in the organs of the body; thus the peculiar odours of the sheep and the goat may be mutually repulsive.

Chirbury, Beckenham, Kent, December 15, I884

\section{A Large Meteor}

A MAGNIFICENT meteor was ob.erved here last night. Its path lay from the west of $\sigma$ Hydræ towards the west of $\eta$ Monocerotis. Its head could not exactly be said to explode but broke up and extended suddenly considerably along its course, emitting a deep red and bluish white light, the latter of a most extraordinary brightness, for a moment quite sufficient to allow print to be discerned. It disappeared very near $I I h$. $19 \mathrm{~m}$. 6s. M.T. Dublin, and left a bluish white trace behind it, which could still with certainty be pcrceived seventeen minutes after the metcor had disappeared.

Birr Castle Observatory, December 23, I884

\section{THE FORMATION OF THE SOLAR SYSTEM}

$\mathrm{THE}$ aspect of the heavens, the appearance of the planets, do not give us the least idea of the solar system. In order to understand it well, we must in imagination quit our world altogether, and remove ourselves to a distance, so as to embrace in one glance the little system of which so ordinary a star as our sun occupies the centre.

Around the sun there move eight primary planets at very unequal distances. Of these planets six have satellites; that is to say, they in their turn are centres of little systems reproducing the solar system in miniature. Thus the Earth has a satellite, the moon; Mars has two, Jupiter four, Saturn eight, Uranus four, and Neptune, the most distant, has one. A striking thing in this system, that which makes it unique, is that the sun turns on its own axis from right to left, and all the planets without exception revolve around it in the same direction, almost in the same plane, that of the rotation of the sun, and describe orbits very nearly circular.

Would not one say that a vast gyratory movement animates all these bodies, and that the secondary systems of the Earth, Mars, Jupiter, \&c., are little whirlpools moving in the primary one? Such was the idea of Descartes. If the solar system does not actually constitute a whirlpool, it was originally formed by a movement of this nature in the nebula which gave it birth.

The sky exhibits here and there a large number of gigantic masses of extremely rarefied matter, like the mists of chaos, without shape, having undergone only that degree of condensation nccessary to create a feeble light. We require usually a powcrful telescope to distinguish them, and then we can see them by thousands in the heavens; these are nebulce.

When you visit an observatory under the escort of an astronomer whom you know, tell him several days beforehand that what you wish is not to gaze at the moon, or the planets and their satellites, or the fixed stars, double ' 'Translation of an article by M. Faye in a recent number of $L$ 'Astronomie. 
or treble, white or coloured, but only to examine the nebulæ of various degrees of condensation. Your wishes being thus indicated, the astronomer will point out to you the most characteristic objects, he will calculate their exact positions, will prepare his most powerful telescope, and then you will be able to make an interesting journey into space.

The nebula of Orion has not a clearly defined form; one region more brilliant than the rest can be distinguished, where the condensation of the chaotic matter is rather far advanced. In all other parts the light is feeble, and one can detect long streamers of matter of which it is impossible to predict the action.

The nebula of Andromeda is one of the most remarkable objects in the heavens. It has an almost geometrical form, and in the centre it exhibits a most distinct condensation.

The nebula of Leo presents nebulous rings in course of formation.

Finally, the curious double nebulæ of Virgo, Aquarius, $\& c$, are evidently very near their ultimate transformation into stars.

It would be easy to multiply the intermediate stages, and to show, for example, some nebulous stars presenting the penultimate phase of this series of transformations, which commences with a feebly luminous mist without shape, and finally arrives at one or many suns variously connected. Needless to say, we are not present at these transformations, but we are like the botanist who in the forest studies the trees in their different degrees of development. Thus the creation of the universe is carried on, so to speak, under our eyes. In the beginning nebula separated out from a universal chaos; in the end, incandescent stars, or other globes so small that we cannot see them, because their formation has produced so little heat that their light is already extinct.

Let us imagine that, owing to some cause of which we shall presently speak, the spirals of a whirling nebula are transformed into nebulous concentric rings, governed by a common movement of rotation. In reality there exist in the heavens objects of this description: for example, the annular nebula in Lyra.

If such as these are rare, it is because they usually do not possess great stability. It is only a transitional form. In reality, in virtue of the differences of linear speed which predominate there, and because of the mutual attraction of their parts, the least cause will lead to eddyings, which, being obliged to follow somewhat the same road with rather different speed, reunite and are lost in a single nebulous mass, where, little by little, all the material of the rings will be absorbed. This nebulous mass, excited by a rotation in the same direction as that of the ring, will in its turn give birth to a planet surrounded by satellites revolving in the same dircction and in the same plane.

We have a serics of nebulous rings, some of which show the eddying condensation which ends in a mass of planets. At the same time the enormous quantity of material which in the midst of the original nebulce was not used up in the rings, has little by little reunited in the middle, very slowly at first, but afterwards very quickly, giving rise to a central globe, a Sun, turning on its own axis in the same direction and in the same plane as the planets.

We thus see how a slow whirling movement, more or less indistinct, would be able to be governed so far as to give rise to these circular rings, all of them concentric and situated in the same plane.

It is necessary and sufficient for this theory that the solar nebula has been, in the first instance, spherical and homogeneous. In such a mass of matter the internal gravity resulting from the attraction of all the molecules varies in a direct ratio with the distance from the centre. The particles or the small bodies which move in such a medium, where the rarity is inconceivable, necessarily describe ellipses or circles round the centre in the sume time, whatever may be their distance from that centre. Thenceforth the existence of rings rotating in one piece, with the same movement, is quite compatible with this condition of gravity, and if a whirling motion has preexisted, some of these spirals, which are not so very different from circles, will have litile by little become transformed into the rings previously described, owing to the small amount of resistance at the centre.

Let us take a step further. In virtue of the force of attraction these rings tend generally to break up and to form a nebulous spherical mass, which in the end contains all the material of the ring. Now these secondary nebulæ must necessarily be endowed with the same direction of rotation as that of the rings. Phenomena exactly like those of the primary nebula will then take place; that is to say, they will resolve themselves into concentric rings, then into $a$ central globe. In their turn, the rings will be condensed into other very small balls-satellites revolving round each planet, always in the same direction, whilst the planet will turn on its own axis exactly in the direction and in the plane of these secondary rings.

It is thus that these things have come about. By a lucky chance some rings of the little secondary system of Saturn have escaped destruction, and have not been formed into satellites. I attribute their existence to the extreme thinness of these rings and to their rapid rotation.

We should now have finished the explanation of the solar system if this system did not offer a striking peculiarity, apparently in complete contradiction with what has preceded. Of the eight large planets revolving round the sun six have satellites, and thus form secondary worlds, exact representations of the solar world which includes them. After what I have said, all the rotations and revolutions ought to be in the same direction, and, what is more, in the "direct" direction. Now in the secondary worlds of the two planets furthest off--those of Uranus and Neptunethe rotations and revolutions of the satellites are in the opposite direction, that is to say, retrograde.

Must we believe that the theory that $I$ have put before you is false? It is not false, but it is incomplete. And here we come to one of the most interesting points in the history of science. Newton and Laplace believed that all the rotations, all the revolutions must be in the same direction. Laplace went further, and applied to this question the theory of probabilities. In working on the planets and satellites as known in his day, his analysis showed that, if a new planet or satellite was discovered, the chances were tens of thousands to one that the revolution of this or that satellite, or the rotation of this or that planet, would be direct, like all the others, and he added that this probability is much greater than that of historical events which we accept with the utmost confidence. The study of the satellites of Uranus, and the discovery of the system of Neptune, however, has at once destroyed this probability, and the celebrated cosmogony of Laplace. This in fact by an ingenious process derives all the planets from the sun, but it can only give to the planets and satellites relations and revolutions in the same direction from one end of the solar system to the other, whilst in fact they are direct in the first half and retrograde in the second.

Let us actually complete our theory. In the primitive nebula, homogeneous and spherical, where the presence of rings revolving round the centre ought not to alter anything in the law of internal gravity, we have seen that this gravity varies in a direct ratio with distance from the centre. But, later, the sun was formed by the reunion of all the matter not wanted for these rings; this has produced an empty space around it. Therefore the law of gravitation in the interior of the system thus modified became quite different. Under the action of the preponderating mass of the sun (that of the rings was not 
the 7ooth part of it) the internal gravity has varied, not in the direct ratio of the distance, but in the inverse ratio of the square of the distance from the centre, and that is the state of things to-day.

In this last case the method of rotation of a ring of diffused matter entirely changes. Let us hasten to say that this alteration does not hinder the ring from existing. Saturn is the proof of it.

But whilst, according to the law of gravity first in operation, the linear velocity of revolution in these rings increased with the distance; according to the second, this velocity on the contrary decreased in the ratio of the square root of this distance.

In the first case, when the ring will have degenerated into a secondary system, that is to say, into a nebula with exterior rings, and finally into a planet with its satellites, the rotation of the planet and the revolution of the satellites will be in the same direction as the movement of the original ring, that is to say, the motion will be "direct." In the second case the secondary system thus formed will be retrograde. ${ }^{1}$

What are we to conclude from this? It is evident that the planets from Mercury to Saturn, included in the central region, were formed according to the first law, when the sun did not yet exist or had not acquired a preponderating mass; and that the planets included in the exterior region, which was by far the larger, were formed when the sun had already come into existence.

If then it should be discovered that Venus had a satellite, its motion would be direct. If a planet were discovered outside Neptune, its rotation and that of its satellites would be retrograde. Here we have at last arrived at a conclusion of the greatest interest: the earth is much older than the sun. If it were otherwise-if, as Laplace would have said, its formation had been long after that of the sun-all would have been changed in the aspect of the skies: the stars would rise in the west and set in the east ; the moon would have a retrograde motion, like the satellites of Uranus and Neptune. Let us add that at that time it was further from the centre than it is now ; for when the matter which was outside the terrestrial orbit had passed over it to be reunited in the interior to orm the sun, as the attraction of the latter gradually preponderated, the revolution of all the planets within the orbit of Uranus was accelerated. These planets approached the sun at the same time that their satellites receded from them.

Finally, the actual state was attained, with the stability which characterises it, when the mass of the sun, having become enormous, could attract nothing more from the original nebulous matter, and had at last created around itself an empty space.

The universe has grown out of chaos, that is to say, out of a mass of matter excessively rare, without shape, occupying a vast space and moving in various directions, in virtue of which this chaotic matter was divided into separate masses. It is by the progressive condensation of these masses of chaotic matter towards certain centres

I Laplace believed that in the nebulous rings derived from the sun (acording to his hypothesis) - rings which will have belonged to the second case as they would be exterior to the sun-the friction of different concentric layers would have had the same effect as what occurs in the atmosphere of a planet, which ends in moving altogether with the central globe. In this way the ring will have taken on the movement of the first form, that is to say a rotation; its outer marginal layers will have had a greater linear speed than that of the layers nearer the centre, and its condensation wil have given place to satellites with direct motion. It is easy to show that this manner of looking is not altogether exact (in proof of this we can point to the rings of Saturn). The layers of an atmosphere press on one another further, the external layers only resist by their inertia to the communica tion of the rotatury movement which tends to establish itself between the central globe and the extreme layers of its atmosphere. But, in a nebulous ring, the concentric layers do not press one on the other as in an atmosphere, for each one moves in virtue of its own speed at its distance from the sun. Further, the retardation of the layers situated near the extreme edge as compared with the internal layers is not due to their inertia, but to the laws of their motion. If then the solar system has been created in accordance with the hypothesis of our great geometrician, all the planets would have revolved round the sun in the direct direction, but their rotations and their satellites would be retrograde. of attraction that the innumerable stars have been formed. Their incandescence comes from the heat developed during the act of their formation. The amount of their heat is limited; they will end by being extinguished.

Amongst all the systems, which are infinitely varied, which have grown out of the condensation of this primary chaos, the solar system may be regarded as a very special case. The primary nebula which gave birth to it was spherical and homogeneous. In separating itself from other portions it had carried with it traces of a slow whirling movement. These motions were soon regulated, thanks to that particular law of internal gravitation resulting from its shape and its homogeneousness. Nebulous rings were thus formed in the same plane long before the appearance of a central condensation. They gave birth to nebulous masses also moving in this plane, in the same direction and in circular orbits, around their common centre.

The secondary systems formed in the same way into these partial nebula can be definitely separated into two categories: those which preceded the formation of the sun, revolving on their own axes in "direct" directions; whilst the secondary systems, the furthest off, formed after the sun, revolve in a retrograde direction. These strange phenomena which are presented by our solar system, are doubtless, by a rare exception in the universe, only the natural consequences of the initial conditions and of the laws of mechanics.

\section{BERZELIUS AND WÜHLER}

THE "Jugenderinnerungen eines Chemikers," which the late Prof. Wohler contributed to the Fournal of the German Chemical Society in 1875 , contains a delightful sketch of the personal relations in which the great German chemist stood to his illustrious master ; and Dr. Hofmann's account of Wöhler's life and works, published in the same journal for 1882 , serves to fill in the details of the picture. The story of Wöhler's visit to Stockholm, of his intercourse with Berzelius, and of the influence which it exerted on the development of his scientific life, are now well known to chemists.

All the papers left by Berzelius are in the possession of the Swedish Academy of Sciences at Stockholm, and among them are the letters which he received from Wöhler. Some time before his death Wöhler presented his letters from Berzelius to the Academy with the injunction that they were not to be published before the close of the present century. Some extracts from the letters of Wöhler, on the publication of which no restriction was made, have recently been given to the world by Dr, Edv. Hjelt of Helsingfors, ${ }^{1}$ from which we may gather some notion of the wealth of material which will be at the disposal of him whose lot it is to write the personal history of the chemistry of this century.

Wöhler's letters to Berzelius extend from I 823 to 1846 , and are 230 in number. In all probability the correspondence was continucd up to the time of Berzelius's death in 1848 , but the letters of the last two years are not contained in the collection. The greater portion of the lelters from Wöhler consist of accounts of his investigations, of discussions of scientific questions, of critical opinions on ncw works and new theories, and of momorabilia of the chemists of the time. Many of the Ietters have reference to the translation of Berzelius's "Jahresbcrichten" and his large "Manual of Chemistry" into German. Now and again we have a gossiping letter, rich in a quiet humour, and occasionally illustrated by quaint characteristic sketches. First in order of time comes Wöhler's application for a place in Berzelius's laboratory, dated July 17, I823, and next is his grateful acknowledg-

x "Bruchstücke aus den Briefen F. Wöhlers an J. J. Berzelius." Herausgegeben von Dr. Edv. Hjelt. (Berlin: Robert Oppenheim, 1884.) 